\title{
Tissular and Cellular Microanalysis using Synchrotron Microprobe Techniques
}

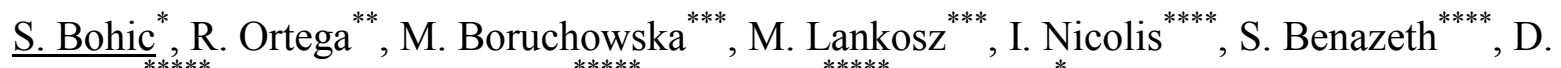
Adamek $^{* * * * *}$, A. Krygowska-Wajs ${ }^{* * * * *}$, B. Tomik ${ }^{* * * *}$, J. Susini ${ }^{*}$

* European Synchrotron Research Facility (ESRF), Beamline ID22-ID21, BP220 38043 Grenoble Cedex 01, France.

** Nuclear chemistry and Bioenvironmental, Bordeaux-Gradignan Nuclear Studies Center BP 120, Le Haut Vigneau, Gradignan, France

*** Department of Radiometric Analyses, Faculty of Physic and Nuclear Techniques, University of Mining and Metallurgy, Krakow, Poland.

**** Laboratoire de Biomathématique, Faculté de Pharmacie, 4 avenue de l'Observatoire 75006 Paris, France

***** Institute of Neurology, Collegium Medicum, Jagiellonian University, Krakow, Poland

Normal and pathological functioning of tissues depend on biochemical processes that proceed at the single cell level and which are in most of the cases not yet fully understood. Investigation of single cell level biochemistry may give us key information to understand the functioning of healthy cells/tissues and the processes turning them into ill ones, thus opening the way to find new medicines/treatments to prevent or slow down some harmful intracellular processes.

It is clear that bulk analysis of cells/tissues can only provide an idea of the main processes but cannot reflect properly the biochemical changes that take place at the single cell level. The most important requirements for microanalysis of single cells are the high spatial resolution $(\sim \mu \mathrm{m})$ and high analytical sensitivity $(\sim \mathrm{ppm})$ for the elements to be investigated. There are several microanalytical tools available (e.g. PIXE, EDX, LAMMA, SIMS, IR or Raman microscopy, microspectrofluorometry) for micron-scale analysis. Nevertheless most of these techniques have some drawbacks related to difficult sample preparation, small depth of analysis, low sensitivity, destruction of the sample, uncertain quantification, which makes their use in single cell research problematical. In order to obtain more complete information the use of several analytical techniques on the very same sample ROI would be desirable, which is very difficult or not fully possible by the above-mentioned techniques. What's more, most of these methods do not provide spectroscopic information on the chemistry at the micron scale and others (IR, Raman...), X ray absorption spectroscopy could be a useful complement.

Due to the high brilliance of synchrotrons of third generation and to the progress in x-ray optics and in synchrotron instrumentation, synchrotron microbeam techniques have a potential to be an important probing method in cellular chemistry [1]. The aim of this presentation is to show through the intracellular $\mathrm{X}$-ray fluorescence imaging studies of cell cultures treated by different metal-based cancer drugs (iododeoxydoxorubicin, gallium, arsenic trioxide) or from pathological tissues (neurodegenerative disorders) that synchrotron based X-ray microfluorescence and micro-XAS (X-absorption spectroscopy) could play an important role in biological and medical research. The Kirkpatrick-Baez focusing mirror recently installed at ID22 lead to major improvements in the size and intensity of the focused beam and made it possible to obtain new results in cellular biology which will be presented. On the basis of the recent results some possible perspectives in biology and medical research can be outlined.

References

[1] S. Bohic et al. Applied Physic Letters 78 (22) (2001) 3544. 


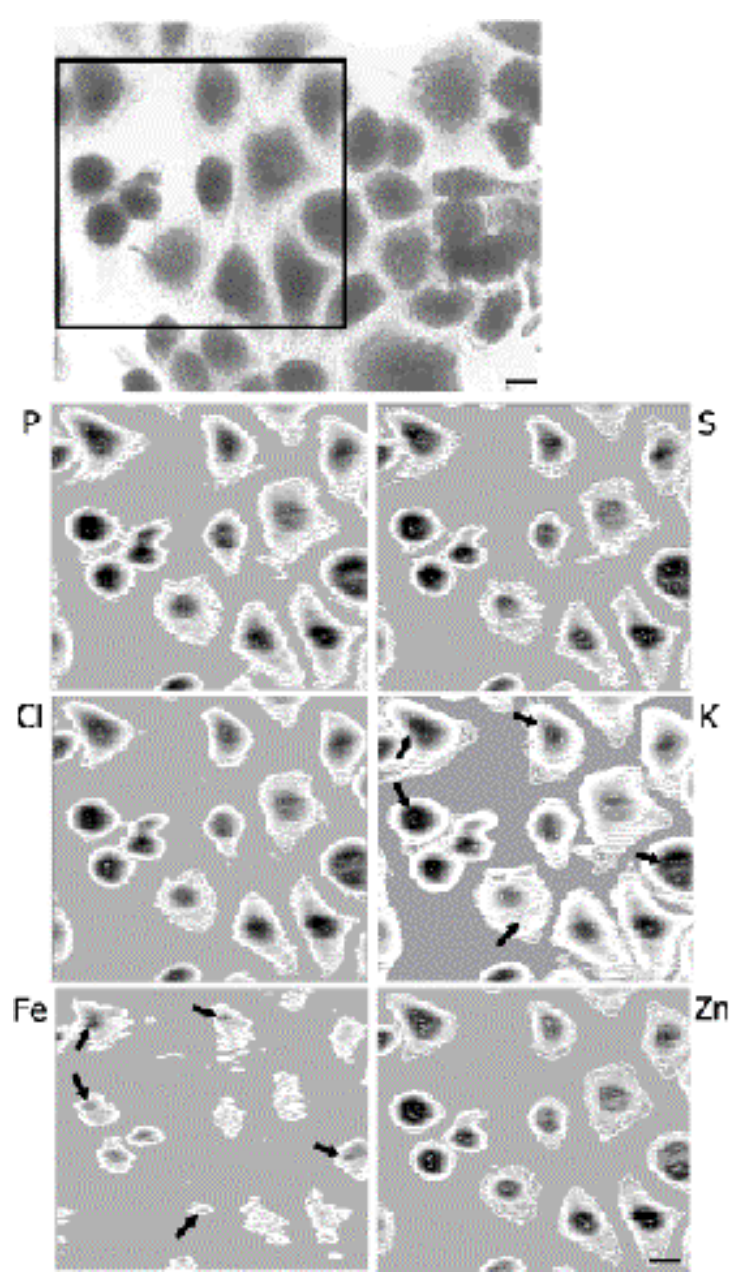

Fig.1: Optical micrograph of dehydrated IGROV-1 cells (top) and corresponding true chemical maps of elements $\mathrm{P}, \mathrm{S}, \mathrm{Cl}, \mathrm{K}, \mathrm{Fe}$ and $\mathrm{Zn}$ obtained using $\mathrm{K}-\mathrm{B}$ focusing system. Background on spectra is completely removed off-line using Axil fitting code in order to define true chemical images of intracellular elements distributions. Element $\mathrm{K}$ is known to be well distributed within cells and constitutes an accurate cell position indicator. $\mathrm{P}, \mathrm{S}$ and $\mathrm{Cl}$, following the $\mathrm{K}$ distribution, are homogenously distributed within cells. $\mathrm{Zn}$ is concentrated in the nucleus, while Fe presents sub-cellular concentrated spots localized in the cytoplasm as indicated by arrows. (Scan size : $100 \times 100 \mu \mathrm{m}^{2}$; Scale bar: $10 \mu \mathrm{m}$ ).
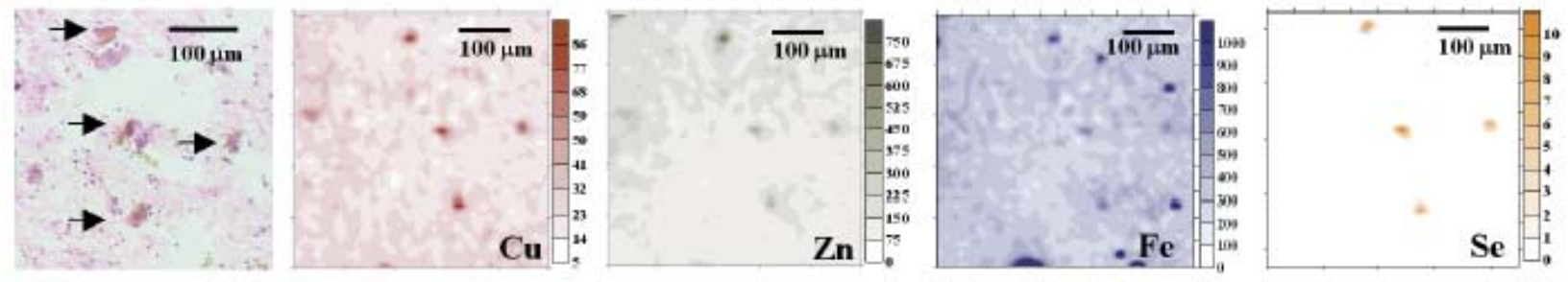

Fig. 2: Substantia nigra - the PD case: (a) microscopic view of scanned area of the tissue; and (b) distribution of selected elements. The arrows show neuron localization. The values on the scales represent intensities in relative units. 